\title{
Pinwheel valence-bond solid and triplet excitations in the two-dimensional deformed kagome lattice
}

\author{
K. Matan ${ }^{1 \star \dagger}$, T. Ono ${ }^{2 \star}$, Y. Fukumoto ${ }^{3 \star}$, T. J. Sato ${ }^{1}$, J. Yamaura ${ }^{4}$, M. Yano ${ }^{2}$, K. Morita $^{2}$ and H. Tanaka ${ }^{2}$
}

Determining ground states of correlated electron systems is fundamental to understanding unusual phenomena in condensed-matter physics. A difficulty, however, arises in a geometrically frustrated system in which the incompatibility between the global topology of an underlying lattice and local spin interactions gives rise to macroscopically degenerate ground states ${ }^{1}$, potentially prompting the emergence of quantum spin states, such as resonating valence bond ${ }^{2-7}$ and valence-bond solid ${ }^{8-11}$ (VBS). Although theoretically proposed to exist in a kagome lattice-one of the most highly frustrated lattices in two dimensions being comprised of cornersharing triangles-such quantum-fluctuation-induced states have not been observed experimentally. Here we report the first realization of the 'pinwheel' VBS ground state in the $S=1 / 2$ deformed kagome lattice antiferromagnet $\mathrm{Rb}_{2} \mathrm{Cu}_{3} \mathrm{SnF}_{12}$ (refs 12,13). In this system, a lattice distortion breaks the translational symmetry of the ideal kagome lattice and stabilizes the VBS state.

Theoretically, significant progress has been made towards understanding the ground state of the quantum $(S=1 / 2)$ kagome lattice antiferromagnet using various approaches. Diverse classes of new states have been proposed; those include a gapless $\mathrm{U}(1)$ Dirac-spin-liquid state $e^{2,5}$, a gapped-spin-liquid state $e^{3,4,6,7}$ and a VBS state $^{8-11}$. One leading approach is based on a quantum dimer model, which was first introduced to describe spin dynamics of singlet pairs (dimers) in high- $T_{\mathrm{c}}$ superconductors ${ }^{14,15}$. On the basis of this model, two kinds of quantum state promptly emerge, namely the VBS and resonating-valence-bond states,7,9-11. The VBS state has long-range dimer-dimer correlations that break the translational symmetry of the ideal kagome lattice with spin-1 singlet-to-triplet excitations, whereas the gapped resonating-valence-bond state, in which different dimer coverings resonate among themselves, has short-range dimer-dimer correlations and topological order with continuum spin- $1 / 2$ excitations (spinons). As these quantum states are non-magnetic and lack static order, one needs to look for their distinct features in magnetic excitations by means of inelastic neutron scattering to distinguish them in a real system.

Experimentally, realizations of these states have been extensively studied in quasi-one dimensional ${ }^{16-18}$ and Shastry-Sutherland twodimensional systems ${ }^{19}$. In contrast, the rarity of model systems has hitherto precluded experimental attempts to observe these states in the kagome lattice. In recent years, the discoveries of the kagome lattice in herbertsmithite ${ }^{20-22}$ and distorted kagome lattice in volborthite ${ }^{23,24}$ have been generating a lot of excitement and debate over their plausible ground states. However, the lack of single crystals and $\mathrm{Zn}-\mathrm{Cu}$ intersite disorder in herbertsmithite hinder the study of these systems. In this work, we present a single-crystal study of a stoichiometrically pure $S=1 / 2$ deformed kagome lattice antiferromagnet $\mathrm{Rb}_{2} \mathrm{Cu}_{3} \mathrm{SnF}_{12}$ with full occupancy of spin-1/2 at the $\mathrm{Cu}$ sites. Combined neutron scattering measurements on a large single crystal and advanced numerical analysis provide the first direct evidence for the pinwheel VBS state.

The magnetic $S=1 / 2 \mathrm{Cu}^{2+}$ ions of $\mathrm{Rb}_{2} \mathrm{Cu}_{3} \mathrm{SnF}_{12}$ reside at the corners of the triangles of the deformed single-layer kagome lattice, and are inside distorted octahedral cages formed by $\mathrm{F}^{-}$ ions. At room temperature, the system crystallizes in the hexagonal $R \overline{3}$ space group with the lattice constants $a=13.917(2) \AA$ and $c=20.356(3) \AA$ (ref. 12; Fig. 1a). The slightly distorted kagome lattice yields four antiferromagnetic exchange interactions (Fig. 1b), which are characterized by different $\mathrm{Cu}^{2+}-\mathrm{F}^{-}-\mathrm{Cu}^{2+}$ bond angles ranging from $124^{\circ}$ for the weakest bond to $138^{\circ}$ for the strongest bond. The system does not exhibit magnetic order down to $1.3 \mathrm{~K}$ (refs 12,13), which is confirmed by our elastic neutron scattering on a powder sample. At low temperatures, magnetic susceptibility rapidly decreases towards zero, a signature of a non-magnetic, spin-singlet $(S=0)$ ground state. Furthermore, low-temperature magnetization sharply rises when the applied field $H$ exceeds a critical field $H_{\mathrm{c}} \approx 20 \mathrm{~T}$, indicative of singlet-pair breaking and closing of a singlet-to-triplet gap. Finite magnetic susceptibility observed at the base temperature of $1.8 \mathrm{~K}$ when the field is applied perpendicular to the crystallographic $c$ axis is attributed to the mixing of the singlet and triplet states through the antisymmetric Dzyaloshinskii-Moriya interaction ${ }^{12}$. Therefore, to a first approximation, the spin Hamiltonian is given by:

$$
\mathcal{H}=\sum_{n n}\left[J_{i j} \mathbf{S}_{i} \cdot \mathbf{S}_{j}+\mathbf{D}_{i j} \cdot \mathbf{S}_{i} \times \mathbf{S}_{j}\right]
$$

where $J_{i j}$ are the nearest-neighbour interactions for four different bonds $J_{1}>J_{2}>J_{3}>J_{4}$ as labelled in Fig. $1 \mathrm{~b}$, and $\mathbf{D}_{i j}=\mathbf{D}_{1}, \mathbf{D}_{2}$, $\mathbf{D}_{3}$ and $\mathbf{D}_{4}$ are the Dzyaloshinskii-Moriya vectors. The in-plane components of these vectors point towards the centre of a triangle and the out-of-plane components alternately point into and out of the kagome plane ${ }^{13}$. The spatial distribution of the non-uniform exchange interactions suggests the stabilization of the pinwheel VBS state ${ }^{25}$ in which singlet dimers are formed between two spins bonded by $J_{1} \sim 20 \mathrm{meV}$ (ref. 12). This pinwheel VBS state is

\footnotetext{
${ }^{1}$ Neutron Science Laboratory, Institute for Solid State Physics, University of Tokyo, 106-1 Shirakata, Tokai, Ibaraki 319-1106, Japan, ${ }^{2}$ Department of Physics, Tokyo Institute of Technology, Meguro-ku, Tokyo 152-8551, Japan, ${ }^{3}$ Department of Physics, Faculty of Science and Technology, Tokyo University of Science, Noda, Chiba 278-8510, Japan, ${ }^{4}$ Institute for Solid State Physics, University of Tokyo, 5-1-5 Kashiwanoha, Kashiwa, Chiba 277-8581, Japan. ${ }^{\dagger}$ Present address: Department of Physics, Faculty of Science, Mahidol University, 272 Rama VI Road, Ratchathewi, Bangkok 10400, Thailand. `e-mail:kmatan@issp.u-tokyo.ac.jp; ono.t.aa@m.titech.ac.jp; yfuku@ph.noda.tus.ac.jp.
} 


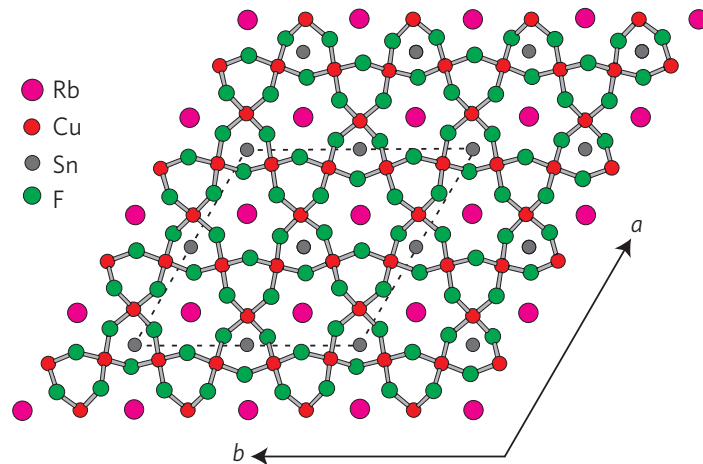

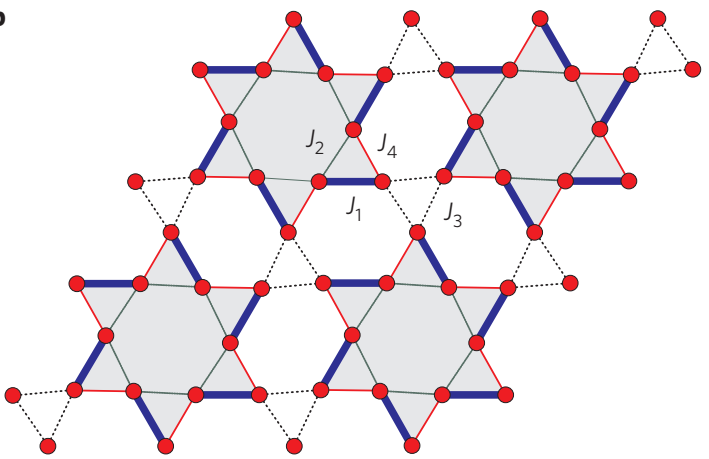

Figure 1 | Crystal structure of $\mathrm{Rb}_{2} \mathrm{Cu}_{3} \mathrm{SnF}_{12} \cdot \mathbf{a}$, A crystal structure in the $a b$ plane shows the connectivity of the $\mathrm{Cu}^{2+}$ ions (red) forming a deformed kagome lattice. b, Dimers (blue bonds) form the pinwheel VBS state. The exchange interactions are labelled as $J_{1}>J_{2}>J_{3}>J_{4}$.

a

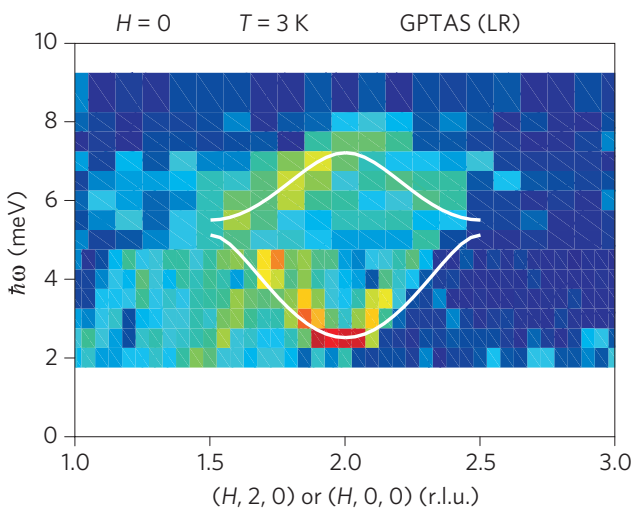

c

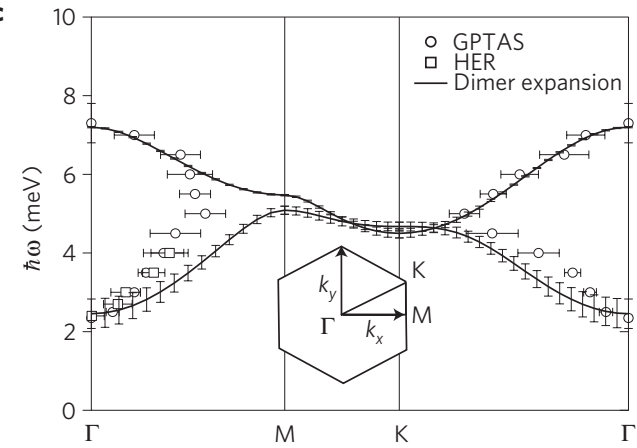

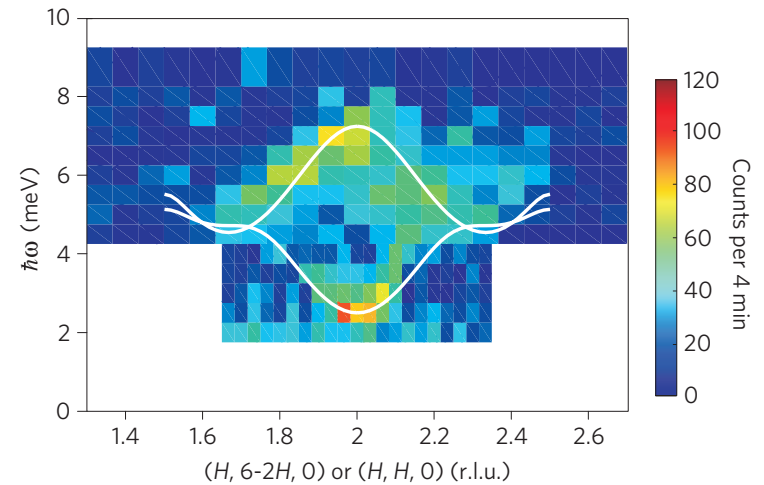

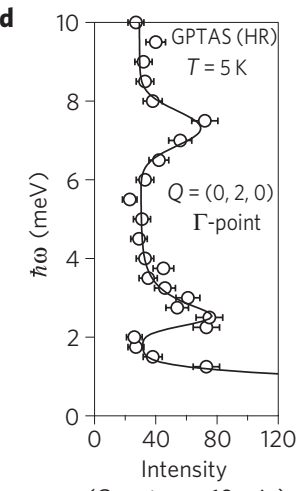

e

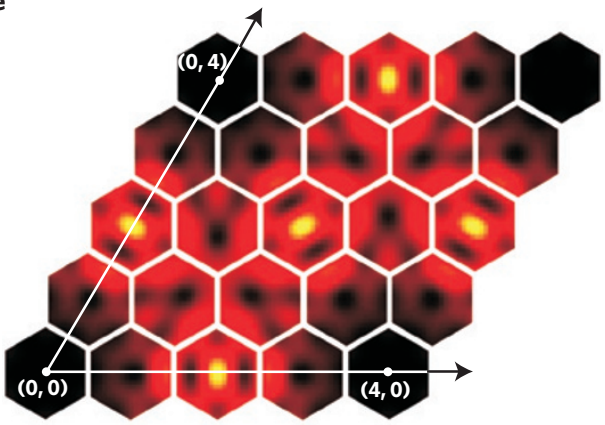

(Counts per $10 \mathrm{~min}$ )

Figure 2 | Energy-momentum contour maps. a,b, Intensity contour maps were measured at $T=3 \mathrm{~K}$ around $(2,0,0)$ and $(2,2,0)$ along two high-symmetry directions, along $k_{x}(\mathbf{a})$ and along $k_{y}(\mathbf{b})$. c, Triplet dispersions were measured along the path shown in the inset. The error bars of the experimental data correspond to three times the statistical error obtained from the fitting (see the Methods section). The solid lines represent the best fits to the dimer series expansion described in the text. The error bars of the calculated curves reflect the differences of various Padé approximations. $\mathbf{d}$, An energy scan at $\mathbf{Q}_{m}=(0,2,0)$ ( $\Gamma$-point) and $T=5 \mathrm{~K}$ shows two spin gaps at 2.35(7) and 7.3(3) meV. The error bars correspond to the counting-statistical error. $\mathbf{e}$, The calculated dynamic structure factor integrated over triplet excitations shows a high scattering intensity around $(2,0,0),(0,2,0)$ and $(2,2,0)$, consistent with the experimental data.

different from the VBS state theoretically proposed for the ideal kagome lattice, because it is stabilized by the lattice distortion and the long-range dimer-dimer correlations do not break the translational symmetry of the lattice. A theoretical study of the $J_{4}$-depleted kagome lattice ${ }^{26}$, which is defined by $J_{1}=J_{2}=J_{3} \equiv J$ and $J_{4} \equiv \alpha J$, shows that the most energetically favoured VBS state proposed for the ideal kagome lattice, which corresponds to $\alpha=1$, is destabilized against this pinwheel state even at $\alpha \sim 0.97$, highlighting the importance of geometrical frustration, which leads to the diversity and closeness of exotic phases in the quantum kagome lattice.
To verify the pinwheel VBS state, we carried out inelastic neutron scattering on a single-crystal sample (see the Methods section). The scattering intensity can be described by the following equation:

$$
I(\mathbf{Q}, \hbar \omega)=\left(\frac{\gamma r_{0}}{2}\right)^{2} f(\mathbf{Q})^{2} S\left(\hbar \omega, \mathbf{q} ; \mathbf{Q}_{m}\right)
$$

where $\left(\gamma r_{0} / 2\right)^{2}=72.65 \times 10^{-3} \mathrm{~b} / \mu_{\mathrm{B}}^{2}, f(\mathbf{Q})$ is a magnetic form factor for $\mathrm{Cu}^{2+}, S\left(\hbar \omega, \mathbf{q} ; \mathbf{Q}_{m}\right)$ is a dynamical structure factor and $\mathbf{Q}=\mathbf{Q}_{m}+\mathbf{q}$. An energy scan at the Brillouin zone centre $\mathbf{Q}_{m}=(0,2,0)$ (Fig. $2 \mathrm{~d}$ ) clearly shows two singlet-to-triplet gaps. The convoluted fit with the resolution function yields the gap 


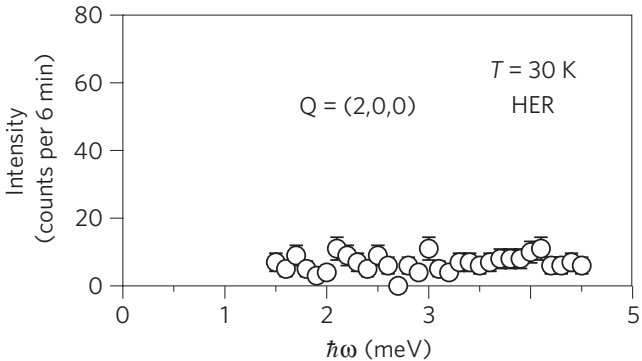

b
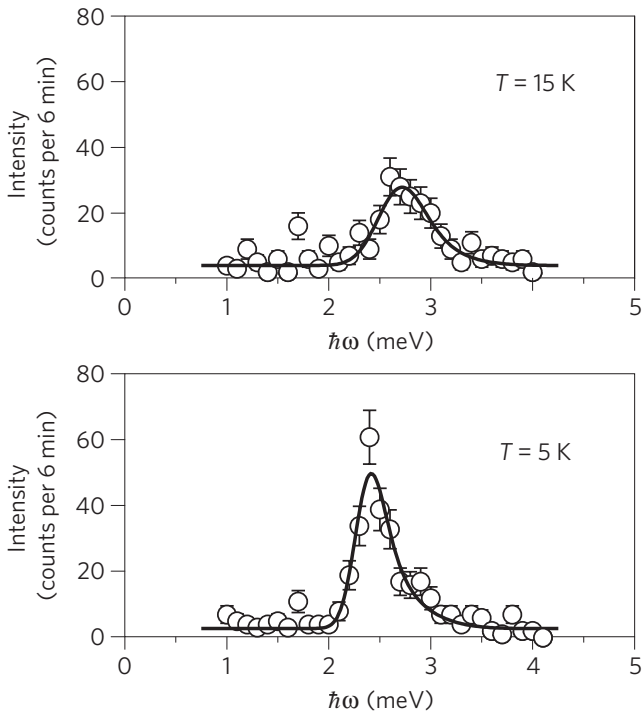

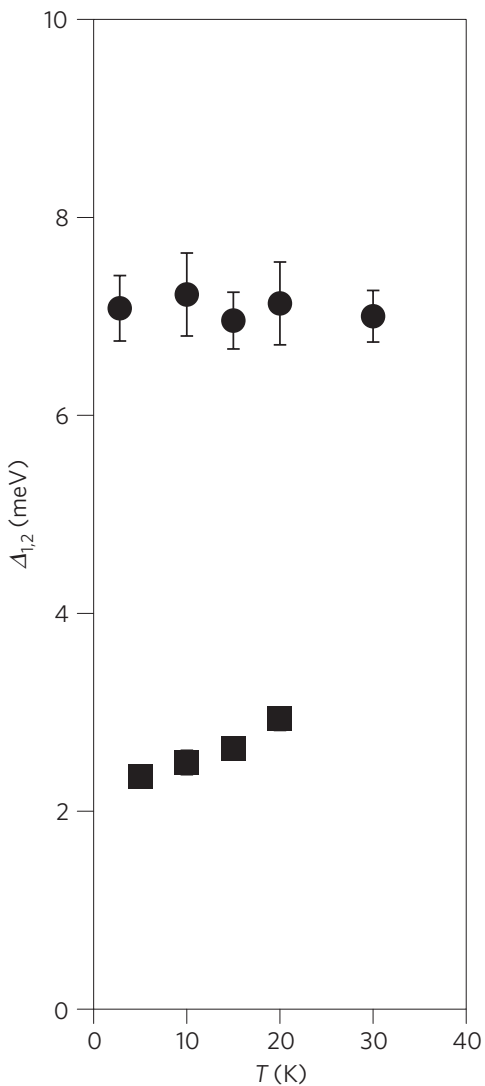

Figure $\mathbf{3}$ | Representative energy scans and temperature dependence. a-c, Energy scans of the lower gap at three temperatures. The error bars correspond to the counting-statistical error. The solid lines denote the fits to the dispersion relation described in the Methods section, convoluted with the instrumental resolution function. Peak widths are resolution limited at the base temperature and become broader with increasing temperatures. d. Temperature dependence of the two triplet gaps; the error bars correspond to three times the statistical error obtained from the fitting.

energies $\Delta_{1}=2.35(7) \mathrm{meV}$ and $\Delta_{2}=7.3(3) \mathrm{meV}$. The thermal renormalization of the spectral weight and damping of the spin excitations (Fig. 3) are typical of triplet modes in an interacting dimer system ${ }^{27}$. In addition, energy scans in an applied magnetic field (Fig. 4) reveal the Zeeman splitting of the lower gap and no splitting of the upper gap. These results demonstrate that, whereas the upper gap is a result of the excitations from the singlet to $S_{\text {tot }}{ }^{z}=0$ triplet states, the lower gap, which is twofold degenerate at zero field, is due to the excitations from the singlet to $S_{\text {tot }}{ }^{z}= \pm 1$ triplet states $\left(S_{\text {tot }}^{z}\right.$ represents a triplet spin state of a single dimer). The Zeeman splitting of the $S_{\text {tot }}{ }^{z}= \pm 1$ triplet gap can be described by the following relation $\Delta_{1, \pm}(H)=\Delta_{1}-g_{ \pm} \mu_{\mathrm{B}} S_{\text {tot }}^{z} H$, where $\mu_{\mathrm{B}}$ is the Bohr magneton. For $S_{\text {tot }}{ }^{z}=1$ and -1 , we obtained $g_{+}=2.58(30)$ and $g_{-}=2.34(20)$, respectively. Extrapolating the $S_{\text {tot }}{ }^{z}=1$ line to intercept the $H$ axis, that is, $\Delta_{1,+}\left(H=H_{c}\right)=0$, yields the critical field $H_{\mathrm{c}} \approx 21 \mathrm{~T}$, consistent with the magnetization data $^{12}$. The magnitude of the Dzyaloshinskii-Moriya vector can be estimated from $|\mathbf{D}| / J_{a v} \sim \Delta g / g \sim 0.2\left(J_{a v} \equiv(1 / 4) \sum_{i} J_{i}\right)$ (ref. 28). Both the Landé $g$ factor and $|D|$ are in good agreement with the values obtained from fitting the susceptibility data to the exact diagonalization for the 12-site kagome cluster ${ }^{12,13}$.

For $\mathrm{Rb}_{2} \mathrm{Cu}_{3} \mathrm{SnF}_{12}$, six dimers in the unit cell give rise to six triplet modes. In the limit $J_{2}-J_{4} \rightarrow 0$, these modes can be characterized by the irreducible representations of the cyclic group $C_{3}$ as four $\mathrm{E}$ and two A localized (flat) modes (see Fig. 6 in ref. 26 and Supplementary Fig. S3). The observed triplet excitations belong to the lowest-energy E mode. The partial lifting of this E-mode triplet degeneracy can be attributed to the Dzyaloshinskii-Moriya interaction. For a single dimer, one can easily show that the Dzyaloshinskii-Moriya interaction raises the energy of the $S_{\text {tot }}{ }^{z}=0$ state, while retaining the degeneracy of the two $S_{\text {tot }}{ }^{z}= \pm 1$ states. To explain the observed large bandwidth and small $S_{\text {tot }}{ }^{z}= \pm 1$ triplet gap, we carried out the dimer series expansion on the pinwheel VBS ground state using the model Hamiltonian given in equation (1). We assume that the strength of the Dzyaloshinskii-Moriya vectors scales with the respective exchange interactions, that is, $d_{z}=D_{i}{ }^{z} / J_{i}$ ( $d_{p}=D_{i}^{p} / J_{i}$ ), where $i=1,2,3$ and 4 for the out-of-plane (in-plane) component. In our first-order dimer expansion (see Supplementary Fig. S3), the out-of-plane component $d_{z}$ of the DzyaloshinskiiMoriya interaction lowers the $S_{\text {tot }}^{z}= \pm 1$ branch at the $\Gamma$-point, which strongly suggests that the observed two branches are the lowest-energy E-mode in the $S_{\text {tot }}{ }^{z}= \pm 1$ and 0 sectors. On the other hand, the in-plane component $d_{p}$ determines only detailed structure of further energy splitting, and thus is omitted in the higher-order calculations. The linked cluster expansion algorithm ${ }^{29}$ was used to obtain a longer series. The lowest-energy triplet excitation spectra were then calculated using the Dlog-Padé approximation up to eighth order in the interdimer exchange and DzyaloshinskiiMoriya interactions. Under the constraints $J_{1}>J_{2}>J_{3}>J_{4}$ and a fixed $J_{a v}$, we obtain the best fit to the excitation spectra (Fig. 2a-c) for $J_{1}=18.6 \mathrm{meV}, J_{2}=0.95 J_{1}, J_{3}=0.85 J_{1}, J_{4}=0.55 J_{1}$ and $d_{z}=0.18$. The agreement between the measured and calculated triplet dispersions verifies the pinwheel VBS state. The integrated dynamic structure factor (Fig. 2e) was calculated to fifth order, assuming equal contributions from two domains, one domain with pinwheel arms in the clockwise direction and the other with pinwheel arms in the anticlockwise direction. These two pinwheel motifs correspond to two equivalent structural domains, which are a mirror image of each other (see Supplementary Fig. S5). The intensity pattern with the periodicity four times the reciprocal lattice vector correlates 

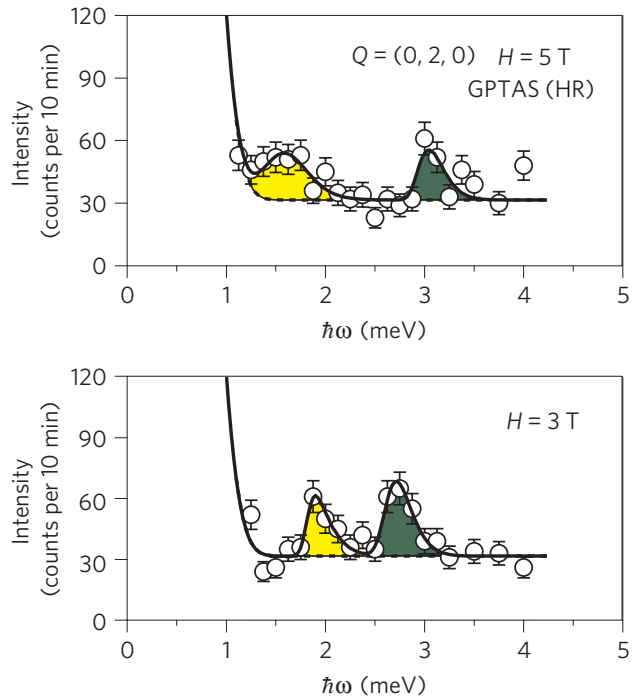

c

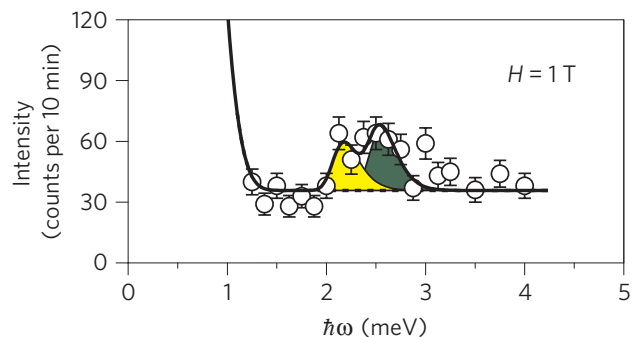

d

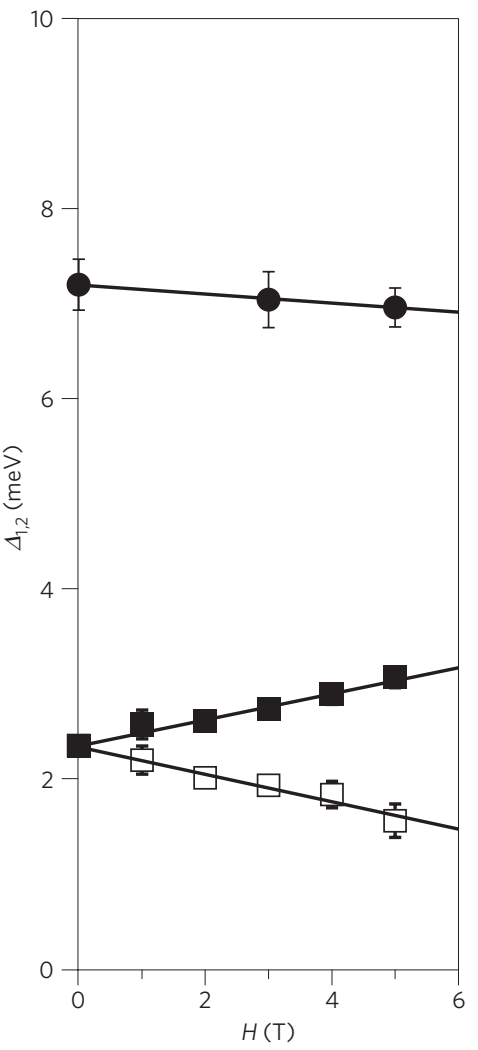

Figure 4 | Triplet excitations in a magnetic field. a-c, Energy scans measured at $T=5 \mathrm{~K}$ show the splitting of the lower gap at three different fields. The error bars correspond to the counting-statistical error. The solid lines denote the fits to the dispersion relation described in the Methods section, convoluted with the instrumental resolution function. The yellow and green shading denotes the $S_{\text {tot }}{ }^{z}=1$ and $S_{\text {tot }}{ }^{z}=-1$ triplet excitations at the $\Gamma$-point, respectively. d, Field dependence of the triplet gaps; the error bars correspond to three times the statistical error obtained from the fitting.

with the intra-dimer distance, which is one fourth of the in-plane lattice constant. The maximum scattering intensity around $(2,0,0)$, $(0,2,0)$ and $(2,2,0)$ also supports the pinwheel VBS state in $\mathrm{Rb}_{2} \mathrm{Cu}_{3} \mathrm{SnF}_{12}$ (see Supplementary Information).

In contrast to triplet excitations in other dimer systems, the highly dispersive triplet bands and the small triplet gap $\left(\Delta_{1} / J_{1} \sim 0.13\right)$ in $\mathrm{Rb}_{2} \mathrm{Cu}_{3} \mathrm{SnF}_{12}$ are due to the strong interdimer interactions, the Dzyaloshinskii-Moriya interaction and the unique pinwheel arrangement of dimers. The bandwidth of the $S_{\text {tot }}{ }^{z}=0$ branch is denominated by $J_{2}-J_{4}$; therefore, the rather small $J_{4} \approx J_{2} / 2$ is essential to explain its relatively large bandwidth. It is also worth noting that the $S_{\text {tot }}{ }^{z}=0$ branch is virtually unaffected by $d_{z}$ and has a gap minimum at the $\mathbf{K}$-point, as predicted by the bond operator mean field theory without the Dzyaloshinskii-Moriya interaction ${ }^{26}$. On the other hand, $d_{z}$ changes the gap minimum of the $S_{\text {tot }}{ }^{z}= \pm 1$ branch from the $\mathbf{K}$-point to the $\Gamma$-point, and significantly lowers the gap energy. If the strength of $d_{z}$ exceeded 0.25 , the gap at the $\Gamma$-point would be closed and magnetic order would instead be observed.

The macroscopically degenerate ground states in the kagome lattice are particularly sensitive to small perturbations, and thereby could inherently give rise to a number of different states in a real system. In $\mathrm{Rb}_{2} \mathrm{Cu}_{3} \mathrm{SnF}_{12}$, our results reveal the pinwheel VBS state that has been suggested to be in close proximity to other phases ${ }^{11,26}$. Recently, we have studied other related compounds, in which a different ground state was observed ${ }^{13}$. In particular, $\mathrm{Cs}_{2} \mathrm{Cu}_{3} \mathrm{SnF}_{12}$ exhibits magnetic order but possesses the low-temperature phase that shares the crystal structure with $\mathrm{Rb}_{2} \mathrm{Cu}_{3} \mathrm{SnF}_{12}$, which suggests similar magnetic interactions. The magnetic order in $\mathrm{Cs}_{2} \mathrm{Cu}_{3} \mathrm{SnF}_{12}$ could, therefore, be ascribed to a small difference in exchange coupling and/or a large Dzyaloshinskii-Moriya interaction ${ }^{30}$. In this case, the former could play a more prominent role in determining the ground state because the Dzyaloshinskii-Moriya interactions in these systems are essentially comparable ${ }^{13}$. In addition, other phases could be obtained by tuning external parameters such as magnetic field and pressure. Further experimental and theoretical studies are desirable to determine precisely the driving mechanism that leads to these different ground states. A more comprehensive understanding of this mechanism could shed some light on many remaining questions regarding the quantum kagome lattice, particularly in relation to the sought-after quantum spin liquid.

\section{Methods}

Experiments. Inelastic neutron scattering experiments were carried out on the HER and GPTAS triple-axis spectrometers operated by the Institute for Solid State Physics, University of Tokyo. Vertically focusing pyrolytic graphite crystals were used to monochromate the incident neutron beam. For the measurements on the HER spectrometer, the scattered neutrons with a fixed final energy of $5 \mathrm{meV}$ were analysed by the central three blades of a seven-blade doubly focused pyrolytic graphite analyser. A cooled Be or oriented pyrolytic graphite crystal filter was placed in the incident beam, and a room-temperature Be filter in the scattered beam to remove higher-order contamination. For the measurements on the GPTAS spectrometer, vertically focused (horizontally flat) pyrolytic graphite crystals were used to analyse scattered neutrons with fixed final energies of 14.7 and $13.7 \mathrm{meV}$. The horizontal collimation sequence of $40^{\prime}-80^{\prime}-$ sample $-80^{\prime}-80^{\prime}$ (LR) or $40^{\prime}-40^{\prime}-$ sample $-40^{\prime}-80^{\prime}$ (HR) was employed with a pyrolytic graphite filter placed in the scattered beam. A single crystal of mass $0.94 \mathrm{~g}$ was mounted in the $h k 0$ and $h 0 l$ zones. The single-crystal sample used in this study was synthesized using the method described in ref. 23. The sample was sealed in an aluminium container in a He gas environment for heat exchange, and cooled by a closed-cycle ${ }^{4} \mathrm{He}$ cryostat. For the measurements in a magnetic field, the sample was aligned in the $h k 0$ zone, and the field was applied along the crystallographic $c$ axis, perpendicular to the kagome plane, using a vertical magnet with a maximum field of $5 \mathrm{~T}$. The sample was then cooled by a liquid ${ }^{4} \mathrm{He}$ cryostat equipped with the magnet.

Data analysis. In Fig. 2a, the measurements were done around $(2,0,0)$ for energies between 2 and $4.5 \mathrm{meV}$, and around $(2,2,0)$ between 4.5 and $9 \mathrm{meV}$. In Fig. $2 \mathrm{~b}$, the measurements were done around $(2,2,0)$ along two equivalent 
high-symmetry directions; for energies between 2 and $4 \mathrm{meV}$, the scans were taken along $(H, H, 0)$, and between 4 and $9 \mathrm{meV}$ along $(H, 6-2 H, 0)$. In Fig. $2 \mathrm{a}$, the triplet branch around $(1.5,0,0)$ could be a result of higher-order corrections to the exchange interactions owing to small atomic displacements as evidenced by weak superlattice reflections; we have ruled out the contribution of other triplet modes (see Supplementary Information). All scans were analysed using equation (2) and an empirical dispersion relation, $\hbar \omega_{\alpha}(\mathbf{q})=\sqrt{\Delta_{\alpha}{ }^{2}-(-1)^{\alpha}\left(v_{\alpha, x}{ }^{2} k_{x}{ }^{2}+v_{\alpha, y}{ }^{2} k_{y}{ }^{2}\right)}$, where $\alpha=1$ or 2 for two non-degenerate branches, and $\mathbf{q}=\left(k_{x}, k_{y}\right)$ is a wave vector away from a Brillouin zone centre $\mathbf{Q}_{m}$. Within the resolution of the instrument of $\sim 1 \mathrm{meV}$ (GPTAS-LR), the out-of-plane dispersion is flat, attesting to the two-dimensionality of the system, and hence we set $v_{\alpha, z}=0$. The observed peaks were fitted with narrow, resolution-limited Lorenztians, convoluted with the resolution function taking into account the empirical dispersion relation. The line shapes are governed by the convolution with the resolution function. The peak positions in units of $\AA^{-1}$ were obtained by fitting $v_{\alpha}$ and an offset $\Delta \mathbf{q}$ (see Supplementary Fig. S2).

Received 22 September 2009; accepted 27 July 2010; published online 12 September 2010

\section{References}

1. Ramirez, A. P. in Handbook of Magnetic Materials Vol. 13 (ed. Buschow, K. H. J.) 423-520 (Elsevier Science, 2001).

2. Hastings, M. Dirac structure, RVB, and Goldstone modes in the kagome antiferromagnet. Phys. Rev. B 63, 014413 (2001).

3. Sachdev, S. Kagome-lattice and triangular-lattice Heisenberg antiferromagnets-Ordering from quantum fluctuations and quantum-disordered ground-states with unconfined bosonic spinons. Phys. Rev. B 45, 12377-12396 (1992).

4. Waldtmann, C. et al. First excitations of the spin $1 / 2$ Heisenberg antiferromagnet on the kagome lattice. Eur. Phys. J. B 2, 501-507 (1998).

5. Ran, Y., Hermele, M., Lee, P. A. \& Wen, X-G. Projected-wave-function study of the spin-1/2 Heisenberg model on the kagome lattice. Phys. Rev. Lett. 98, 117205 (2007)

6. Zeng, C. \& Elser, V. Quantum dimer calculations on the spin-1/2 kagome Heisenberg-antiferromagnet. Phys. Rev. B 51, 8318-8324 (1995).

7. Misguich, G., Serban, D. \& Pasquier, V. Quantum dimer model on the kagome lattice: Solvable dimer-liquid and Ising gauge theory. Phys. Rev. Lett. 89, 137202 (2002).

8. Marston, J. B. \& Zeng, C. Spin-Peierls and spin-liquid phases of kagome quantum antiferromagnets. J. Appl. Phys. 69, 5962-5964 (1991).

9. Nikolic, P. \& Senthil, T. Physics of low-energy singlet states of the kagome lattice quantum Heisenberg antiferromagnet. Phys. Rev. B 68, 214415 (2003)

10. Singh, R. R. P. \& Huse, D. A. Ground state of the spin-1/2 kagome-lattice Heisenberg antiferromagnet. Phys. Rev. B 76, 180407(R) (2007).

11. Yang, B-J., Kim, Y. B., Yu, J. \& Park, K. Spin triplet excitations for a valence bond solid on the kagome lattice. Phys. Rev. B 77, 224424 (2008).

12. Morita, K. et al. Singlet ground state and spin gap in $\mathrm{S}=1 / 2$ kagome antiferromagnet $\mathrm{Rb}_{2} \mathrm{Cu}_{3} \mathrm{SnF}_{12}$. J. Phys. Soc. Jpn 77, 043707 (2008).

13. Ono, T. et al. Magnetic susceptibilities in a family of $S=1 / 2$ kagome antiferromagnets. Phys. Rev. B 79, 174407 (2009).

14. Rokhsar, D. \& Kivelson, S. Superconductivity and the quantum hard-core dimer gas. Phys. Rev. Lett. 61, 2376-2379 (1988).
15. Moessner, R., Sondhi, S. L. \& Fradkin, E. Short-ranged resonating valence bond physics, quantum dimer models, and Ising gauge theories. Phys. Rev. $B$ 65, 024504 (2002).

16. Fujita, O., Akimitsu, J., Nishi, M. \& Kakurai, K. Evidence for a singlet-triplet transition in spin-Peierls system $\mathrm{CuGeO}_{3}$. Phys. Rev. Lett. 74, 1677-1680 (1995).

17. Nikuni, T., Oshikawa, M., Oosawa, A. \& Tanaka, H. Bose-Einstein condensation of dilute magnons in $\mathrm{TlCuCl}_{3}$. Phys. Rev. Lett. 84, 5868-5871 (2000).

18. Lake, B., Tennant, D., Frost, C. D. \& Nagler, S. E. Quantum criticality and universal scaling of a quantum antiferromagnet. Nature Mater. 4, 329-334 (2005).

19. Kageyama, H. et al. Exact dimer ground state and quantized magnetization plateaus in the two-dimensional spin system $\mathrm{SrCu}_{2}\left(\mathrm{BO}_{3}\right)_{2}$. Phys. Rev. Lett. 82, 3168-3171 (1999)

20. Helton, J. S. et al. Spin dynamics of the spin-1/2 kagome lattice antiferromagnet $\mathrm{ZnCu}_{3}(\mathrm{OH})_{6} \mathrm{Cl}_{2}$. Phys. Rev. Lett. 98, 107204 (2007).

21. Mendels, P. et al. Quantum magnetism in the paratacamite family: Towards an ideal kagome lattice. Phys. Rev. Lett. 98, 077204 (2007).

22. Lee, S-H. et al. Quantum-spin-liquid states in the two-dimensional kagome antiferromagnets $\mathrm{Zn}_{x} \mathrm{Cu}_{4-x}(\mathrm{OD})_{6} \mathrm{Cl}_{2}$. Nature Mater. 6, 853-857 (2007).

23. Bert, F. et al. Ground state of the kagome-like $S=1 / 2$ antiferromagnet volborthite $\mathrm{Cu}_{3} \mathrm{~V}_{2} \mathrm{O}_{7}(\mathrm{OH})_{2} \cdot 2 \mathrm{H}_{2} \mathrm{O}$. Phys. Rev. Lett. 95, 087203 (2005).

24. Yoshida, M., Takigawa, M., Yoshida, H., Okamoto, Y. \& Hiroi, Z. Phase diagram and spin dynamics in volborthite with a distorted kagome lattice. Phys. Rev. Lett. 103, 077207 (2009).

25. Syromyatnikov, A. V. \& Maleyev, S. V. Hidden long-range order in kagome Heisenberg antiferromagnets. Phys. Rev. B 66, 132408 (2002).

26. Yang, B-J. \& Kim, Y. B. Valence bond solid phases on deformed kagome lattices: Application to $\mathrm{Rb}_{2} \mathrm{Cu}_{3} \mathrm{SnF}_{12}$. Phys. Rev. B 79, 224417 (2009).

27. Rüegg, C. et al. Quantum statistics of interacting dimer spin systems. Phys. Rev. Lett. 95, 267201 (2005).

28. Moriya, T. Anisotropic superexchange interaction and weak ferromagnetism. Phys. Rev. 120, 91-98 (1960).

29. Oitmaa, J., Hamer, C. \& Zhang, W. Series Expansion Methods for Strongly Interacting Lattice Models (Cambridge Univ. Press, 2006).

30. Cépas, O., Fong, C. M., Leung, P. W. \& Lhuillier, C. Quantum phase transition induced by Dzyaloshinskii-Moriya interactions in the kagome antiferromagnet. Phys. Rev. B 78, 140405(R) (2008).

\section{Acknowledgements}

We thank M. Nishi, C. Broholm, K. Iida and M. Kofu for useful discussions. The work at Tokyo Institute of Technology was supported by a Grant-in-Aid for Scientific Research from JSPS and a Global COE Program funded by MEXT Japan.

\section{Author contributions}

K. Matan, T.O. and T.J.S. carried out neutron scattering measurements. J.Y. carried out X-ray diffraction measurements. Y.F. carried out numerical calculations. T.O., M.Y., K. Morita and H.T. contributed to sample synthesis and characterization.

\section{Additional information}

The authors declare no competing financial interests. Supplementary information accompanies this paper on www.nature.com/naturephysics. Reprints and permissions information is available online at http://npg.nature.com/reprintsandpermissions. Correspondence and requests for materials should be addressed to K.M., T.O. or Y.F. 